\title{
Mimetic Einstein-Cartan-Sciama-Kibble (ECSK) gravity
}

\author{
Fernando Izaurieta, ${ }^{a}$ Perla Medina, ${ }^{a, b, c}$ Nelson Merino, ${ }^{d}$ Patricio Salgado ${ }^{d}$ \\ and Omar Valdivia ${ }^{d, e, 1}$ \\ ${ }^{a}$ Departamento de Fúsica, Universidad de Concepción, \\ Casilla 160-C, Concepción, Chile \\ ${ }^{b}$ Universidad Austral de Chile (UACh), Campus Isla Teja, \\ Ed. Emilio Pugin, Valdivia, Chile \\ ${ }^{c}$ Centro de Estudios Cientificos (CECs), \\ Avenida Arturo Prat 514, Valdivia, Chile \\ ${ }^{d}$ Instituto de Ciencias Exactas y Naturales (ICEN), Facultad de Ciencias, \\ Universidad Arturo Prat, Iquique, Chile \\ ${ }^{e}$ Institute of Space Sciences (IEEC-CSIC), \\ C. Can Magrans s/n, 08193 Cerdanyola del Valles (Barcelona), Spain \\ E-mail: fizaurie@udec.cl, perlamedina@udec.cl, nemerino@unap.cl, \\ patsalgado@unap.cl, ovaldivi@unap.cl
}

ABSTRACT: In this paper, we formulate the Mimetic theory of gravity in first-order formalism for differential forms, i.e., the mimetic version of Einstein-Cartan-Sciama-Kibble (ECSK) gravity. We consider different possibilities on how torsion is affected by Weyl transformations and discuss how this translates into the interpolation between two different Weyl transformations of the spin connection, parameterized with a zero-form parameter $\lambda$. We prove that regardless of the type of transformation one chooses, in this setting torsion remains as a non-propagating field. We also discuss the conservation of the mimetic stress-energy tensor and show that the trace of the total stress-energy tensor is not null but depends on both, the value of $\lambda$ and spacetime torsion.

Keywords: Classical Theories of Gravity, Cosmology of Theories beyond the SM

ArXiv EPRINT: 2007.07226

\footnotetext{
${ }^{1}$ Corresponding author.
} 


\section{Contents}

1 Introduction 1

2 Review of mimetic gravity 3

3 ECSK gravity and first order formalism 4

4 Conformal Riemann-Cartan structure $\quad 6$

5 Mimetic ECSK gravity $\quad 8$

$\begin{array}{llr}5.1 \text { Mimetic field equations } & 9\end{array}$

$\begin{array}{lll}5.2 \text { Conservation laws } & 11\end{array}$

6 The trace of the stress-energy tensor, torsion and $\lambda \quad 13$

$\begin{array}{lll}7 & \text { Summary \& comments } & 14\end{array}$

\section{Introduction}

General Relativity (GR) is a classical field theory describing the gravitational interaction through the Einstein field equations. Remarkably, it has proven success in a wide range of phenomena [1], including black-holes as realistic astrophysical objects [2] and the existence of gravitational waves [3-5]. Another significant development of GR lies in the context of cosmology, where extending Einstein's field equations, by the inclusion of an early inflationary stage and a cold dark matter contribution, is in good agreement with observational data [6]. This fact is somehow dramatic since we know very little about the nature of dark matter, apart from the fact that it does not appear to interact with the electromagnetic field. For this reason, the problem of identifying dark matter candidates attracts attention from both cosmology and particle physics. There are several dark matter candidates: weakly interacting massive particles, sterile neutrinos, axions, cold massive halo objects, and primordial black holes [7-9]. Nevertheless, despite many experiments to directly detect and study dark matter particles being actively undertaken, none have succeeded.

Given the difficulties for the standard cosmology models to describe the nature of dark matter (See, for instance, [10]), there has been a popular trend for considering modified gravity models [11-16]. Most of these models attempt to account for all observations without invoking supplemental non-baryonic matter, but observational constraints make it hard to create a consistent model of modified gravity still compatible with GR's principles.

Another exciting direction is to consider GR beyond the limits of Riemannian geometry. The canonical model generalizing GR is the Einstein-Cartan-Sciama-Kibble (ECSK) gravity. This modification came firstly with the works of Elie Cartan in 1922, before the discovery of spin. However, Cartan's model did not bring much attention until the late 1950s, 
where Sciama and Kibble rediscovered Cartan's results $[17,18]$. The main feature of ECSK gravity is that it accounts for spacetime torsion [19]. In ECSK, quantum mechanical spin acts as the source of torsion, ${ }^{1}$ in the same way as energy is a source of curvature [21, 22]. Consequently, torsion could have been relevant in the early universe because of its extremely high fermion-densities [23-30]. Another interesting observation in standard ECSK is that the torsion two-form does not propagate in a vacuum, and it interacts very weakly with Standard Model fermions (See Chap. 8.4 of ref. [31] and refs. [32-34]). Thus, torsion might potentially be a component of dark matter [35-40].

More recently, in [41, 42], Chamseddine and Mukhanov have considered a different approach for addressing dark matter with the Mimetic gravity theory. This model shows that the conformal degree of freedom of the gravitational field becomes dynamical even in the absence of matter. This extra degree of freedom corresponds to the mimetic field's energy density, which mimics the stress-energy tensor of extra pressureless dust without needing dark matter particles. Moreover, ref. [43] discusses whether mimetic cosmology can create late-time acceleration and the inflationary stage of the universe. Besides, many authors have considered different aspects of mimetic gravity during the last years with exciting results. Some examples are black holes [44-54], black strings [55], and braneworld scenarios [56-61], among others. For a more exhaustive survey, see [62-103] and references therein.

In this paper, we pursue constructing a mimetic theory of gravity in first-order formalism for differential forms. For a sufficiently general family of Weyl transformations for the affine connection, the resulting theory is equivalent to "mimicking" the ECSK model. For the construction, we assume that the vierbein one-form $e^{a}(x)$ and the spin connection one-form $\omega^{a b}(x)$ describe independent metric and affine properties of the spacetime. We consider diverse ways of generalizing Weyl transformations for a Riemann-Cartan geometry with independent vierbein and spin connection, following a similar approach to [104]. Remarkably, none of these generalizations generate a propagating torsion field. Therefore, in this setting, only a non-vanishing spin tensor can be a source of torsion. When using scalar fields, non-minimal couplings and higher derivatives terms seem to be the way of creating a propagating torsion (See, for instance, $[105,106]$ ). When considering generalized conformal invariance on Riemann-Cartan geometry, the stress-energy tensor's trace, which usually vanishes for conformally invariant theories of gravity, has a non-zero value depending on torsion and on a parameter which characterizing Weyl transformations for the spin connection.

This paper organization is the following. In section 2, we summarize the main aspects of mimetic gravity. In section 3, we revisit ECSK gravity, and we give a brief description of Cartan's first order formalism for differential forms. In section 4, we introduce some useful mathematical tools to describe conformal structures in Cartan's first-order formalism. In section 5, we derive the equations of mimetic gravity in first-order formalism, show how these correspond to the mimetic ECSK model, and analyze the conservation law for the

\footnotetext{
${ }^{1}$ It is important to stress that with spin, we refer only to intrinsic quantum mechanical spin, and we should not confuse this with the angular momentum density. This natural confusion has already led to some mistakes in the literature, see ref. [20].
} 
mimetic stress-energy tensor. Finally, in section 6, we discuss the stress-energy tensor's trace and its dependency on torsion and conformal parameter $\lambda$. The paper concludes in 7 with a summary and comments regarding some possible cosmological applications.

\section{Review of mimetic gravity}

Mimetic Gravity was first introduced by A. Chamseddine and V. Mukhanov as a theory of gravity which naturally exhibits conformal symmetry as internal degree of freedom [41]. Let $M_{4}$ be a four dimensional spacetime and let us consider a physical metric $g_{\mu \nu}$, with Lorentz signature $(-,+,+,+)$, depending on an auxiliary metric $\bar{g}_{\mu \nu}$ and a scalar field $\phi$, namely

$$
g_{\mu \nu}=-\bar{g}^{\alpha \beta} \partial_{\alpha} \phi \partial_{\beta} \phi \bar{g}_{\mu \nu} .
$$

The metric $g_{\mu \nu}$ is invariant with respect to Weyl transformations of the auxiliary metric $\bar{g}_{\mu \nu}$, i.e., it remains unchanged after rescaling

$$
\bar{g}_{\mu \nu} \rightarrow \Omega^{2}(x) \bar{g}_{\mu \nu} .
$$

Additionally, it follows from (2.1) that

$$
g^{\alpha \beta} \partial_{\alpha} \phi \partial_{\beta} \phi=-1
$$

The resultant new degree of freedom associated with the transformation (2.1) represents the longitudinal mode of gravity which is excited even in the absence of any matter field configurations.

The canonical action of GR is rewritten by considering the physical metric $g_{\mu \nu}$ as function of the scalar field $\phi$ and the auxiliary metric $\bar{g}_{\mu \nu}$

$$
S=\frac{1}{c} \int \mathrm{d}^{4} x \sqrt{-g\left(\bar{g}_{\mu \nu}, \phi\right)}\left[\frac{1}{\kappa_{4}}\left(\frac{1}{2} R\left(\bar{g}_{\mu \nu}, \phi\right)-\Lambda\right)+L_{m}\right],
$$

where $\kappa_{4}=\frac{8 \pi G}{c^{4}}, R$ is the Ricci scalar constructed from $g_{\mu \nu}$ and $L_{m}$ stands for the matter Lagrangian. The action (2.4) is invariant under Weyl transformations because it only depends on $g_{\mu \nu}$ which is conformally invariant under (2.2). The resulting dynamics can be directly obtained by starting from the variation of (2.4) with respect to the physical metric $g_{\mu \nu}$, then expressing $\delta g_{\mu \nu}$ in terms of $\delta \bar{g}_{\mu \nu}$ and $\delta \phi$ and assuming that the last two are independent. Thus, field equations read

$$
\begin{aligned}
G^{\mu \nu}-\kappa_{4} \mathcal{T}^{\mu \nu}+\left(G-\kappa_{4} \mathcal{T}\right) g^{\lambda \mu} g^{\sigma \nu} \partial_{\lambda} \phi \partial_{\sigma} \phi & =0 \\
\nabla_{\mu}\left[\left(G-\kappa_{4} \mathcal{T}\right) \partial^{\mu} \phi\right] & =0
\end{aligned}
$$

where $G_{\mu \nu}=R_{\mu \nu}-\frac{1}{2} g_{\mu \nu} R+\Lambda g_{\mu \nu}$ is the Einstein tensor, $\mathcal{T}_{\mu \nu}$ the energy momentum tensor, and $G, \mathcal{T}$ denote their respective traces. The dynamics given in (2.5) and (2.6) departs from pure GR.

In ref. [107], an equivalent formulation of Mimetic Gravity has been proposed where, instead of introducing $\phi$ through the reparametrization (2.4), the physical metric $g_{\mu \nu}$ is 
directly used together with a constrained scalar field, enforcing (2.3) through a Lagrange multiplier. Taking the trace in (2.5), direct calculation shows

$$
\left(G-\kappa_{4} \mathcal{T}\right)\left(1+g^{\alpha \beta} \partial_{\alpha} \phi \partial_{\beta} \phi\right)=0 .
$$

This last equation is automatically satisfied by the constraint $(2.3)$ even for $G \neq \kappa_{4} \mathcal{T}$. From this point of view, even in absence of matter, the gravitational field equations have non-trivial solutions for the conformal mode. To understand this extra degree of freedom, let us rewrite eq. (2.5) as

$$
G^{\mu \nu}=\kappa_{4}\left(\mathcal{T}^{\mu \nu}+\overline{\mathcal{T}}^{\mu \nu}\right)
$$

where

$$
\overline{\mathcal{T}}^{\mu \nu}=\left(\mathcal{T}-\frac{G}{\kappa_{4}}\right) g^{\mu \alpha} g^{\nu \beta} \partial_{\alpha} \phi \partial_{\beta} \phi
$$

Now compare this expression with the energy momentum tensor for a perfect fluid

$$
\mathcal{T}^{\mu \nu}=\frac{1}{c^{2}}(\rho+p) u^{\mu} u^{\nu}-p g^{\mu \nu},
$$

where $\rho$ is the energy density, $p$ is the pressure and $u^{\mu}$ is the four-velocity which satisfies $\frac{1}{c^{2}} u^{\lambda} u_{\lambda}=-1$. Setting $p=0$ and making the following identification

$$
\begin{aligned}
\rho & =\left(\mathcal{T}-\frac{G}{\kappa_{4}}\right), \\
u^{\mu} & =c g^{\mu \alpha} \partial_{\alpha} \phi,
\end{aligned}
$$

the energy momentum tensor (2.10) becomes equivalent to $\overline{\mathcal{T}}^{\mu \nu}$. Thus, the extra degree of freedom mimics the potential motions of dust with energy density $\left(\mathcal{T}-\frac{G}{\kappa_{4}}\right)$, and the scalar field plays the role of a velocity potential. In absence of matter this energy density is proportional to $G=4 \Lambda-R$, which does not vanish for generic solutions. Note that the normalization condition for the four velocity $u^{\mu}$ and the conservation law for $\overline{\mathcal{T}}^{\mu \nu}$, are equivalent to (2.3) and (2.6), respectively.

\section{ECSK gravity and first order formalism}

So far we have used Greek indices $\mu, \nu, \ldots$ to denote tensor components in the coordinate basis. From now on, we use lower case Latin indices $a, b, \ldots$ for tensors defined in Lorentz (orthonormal) basis. We denote by $\Omega^{p}\left(M_{4}\right)$ to the set of differential $p$-forms defined over $M_{4}$.

At a particular point $P \in M_{4}$, the vierbein components $e^{a}{ }_{\mu}(x)$ are determined through the relation

$$
g_{\mu \nu}=\eta_{a b} e^{a}{ }_{\mu} e^{b}{ }_{\nu}
$$

where $\eta_{a b}$ is the Minkowski metric. In terms of $e^{a}{ }_{\mu}(x)$ we define the vierbein $e^{a}=e^{a}{ }_{\mu}(x) \mathrm{d} x^{\mu}$ as the set of one-forms $\Omega^{1}\left(M_{4}\right)$. The vierbein contains all the information on the metric. Moreover, the affine properties of geometry are described by the one-form spin connection 
$\omega^{a b}=\omega^{a b}{ }_{\mu}(x) \mathrm{d} x^{\mu}$, which is anti-symmetric provided the metricity condition $\nabla_{\lambda} g_{\mu \nu}=0$. Additionally, it relates to the affine connection $\Gamma_{\mu \nu}^{\lambda}$ through the vierbein postulate

$$
\partial_{\mu} e^{a}{ }_{\nu}+\omega^{a}{ }_{b \mu} e^{b}{ }_{\nu}-\Gamma_{\mu \nu}^{\lambda} e_{\lambda}^{a}=0 .
$$

The covariant derivative of the vierbein is defined as the two-form torsion $T^{a}=\mathrm{D} e^{a}$ where

$$
\mathrm{D} e^{a}=\mathrm{d} e^{a}+\omega^{a}{ }_{b} \wedge e^{b} .
$$

Unlike $\mathrm{d}^{2}=0$, higher order covariant derivatives of the vierbein does not vanish. In fact, direct calculation shows $\mathrm{D} T^{a}=R_{b}^{a} \wedge e^{b}$ where

$$
R^{a b}=\mathrm{d} \omega^{a b}+\omega^{a}{ }_{c} \wedge \omega^{c b},
$$

is the Lorentz two-form curvature which transforms covariantly under local Lorentz transformations.

The spin connection can also be decomposed in a torsion-free part $\stackrel{\circ}{\omega}^{a b}$ satisfying

$$
\mathrm{d} e^{a}+\stackrel{\circ}{\omega}_{b}^{a} \wedge e^{b}=0,
$$

and a second rank anti-symmetric one-form $\kappa^{a b}$, usually called the contorsion or contortion. An important observation is that $\stackrel{\circ}{\omega}^{a b}$ is completely determined in terms of the vierbein. Therefore, all the affine degree of freedom are encoded into the contorsion

$$
\kappa^{a b}=\omega^{a b}-\stackrel{\circ}{\omega}^{a b},
$$

and consequently $T^{a}=\kappa^{a}{ }_{b} \wedge e^{b}$. In terms of this splitting, the Lorentz curvature is given by

$$
R^{a b}=\stackrel{\circ}{R}^{a b}+\stackrel{\circ}{\mathrm{D}} \kappa^{a b}+\kappa^{a}{ }_{c} \wedge \kappa^{c b},
$$

where $\stackrel{\circ}{R}^{a b}=\mathrm{d} \stackrel{\circ}{\omega}^{a b}+\stackrel{\circ}{\omega}_{c}^{a} \wedge \stackrel{\circ}{\omega}^{c b}$ is the Riemann curvature two-form and $\stackrel{\circ}{\mathrm{D}}$ stands for the covariant derivative with respect to the torsion-free part of the connection $\stackrel{\circ}{\omega}^{a b}$.

There are many theories leading to non-vanishing torsion; see for instance refs. [33, 3639, 105, 108-115], and for the most general case, Poincaré Gauge Theory, see [21, 116120]). However, the closest to standard General Relativity is Einstein-Cartan-KibbleSciama (ECSK) gravity [121-127]. In this framework, geometry also depends of the spin tensor of matter, but its physical effects would be noticeable only for densities much higher than nuclear density [128]. Moreover, it has been shown that torsion prevents cosmological singularities [129-134], gives rise to a new universe from a collapse [23, 135, 136], and introduce an effective ultraviolet cutoff in a quantum field theory for fermions [128].

In differential form language, the ECSK four-form Lagrangian is given by

$$
\mathcal{L}_{\mathrm{ECSK}}=\mathcal{L}_{\mathrm{G}}(e, \omega)+\mathcal{L}_{\mathrm{M}}(e, \omega, \varphi),
$$

where

$$
\mathcal{L}_{\mathrm{G}}=\frac{1}{4 \kappa_{4}} \epsilon_{a b c d}\left(R^{a b}-\frac{\Lambda}{3 !} e^{a} \wedge e^{b}\right) \wedge e^{c} \wedge e^{d},
$$


is the four-form Lagrangian for geometry and $\mathcal{L}_{\mathrm{M}}$ is the four-form Lagrangian for matter fields. Up to boundary terms, variation of the action functional $S=\frac{1}{c} \int_{M_{4}} \mathcal{L}_{\mathrm{ECSK}}$ reads

$$
\delta S=\frac{1}{c} \int_{M_{4}} \frac{1}{\kappa_{4}}\left(\frac{1}{2} \delta \omega^{a b} \wedge \mathcal{W}_{a b}+\mathcal{E}_{d} \wedge \delta e^{d}\right),
$$

with

$$
\begin{aligned}
\mathcal{E}_{d} & =\epsilon_{a b c d}\left(\frac{1}{2} R^{a b}-\frac{\Lambda}{3 !} e^{a} \wedge e^{b}\right) \wedge e^{c}-\kappa_{4} * \mathcal{T}_{d}, \\
\mathcal{W}_{a b} & =\epsilon_{a b c d} T^{c} \wedge e^{d}-\kappa_{4} * \sigma_{a b} .
\end{aligned}
$$

Here, the relations

$$
\begin{aligned}
\delta_{e} \mathcal{L}_{\mathrm{M}}^{(4)} & =-* \mathcal{T}_{d} \wedge \delta e^{d}, \\
\delta_{\omega} \mathcal{L}_{\mathrm{M}}^{(4)} & =-\frac{1}{2} \delta \omega^{a b} \wedge * \sigma_{a b},
\end{aligned}
$$

implicitly define the stress-energy one-form $\mathcal{T}^{a}=\mathcal{T}^{a}{ }_{\mu} \mathrm{d} x^{\mu}$ and the spin tensor 1-form $\sigma^{a b}=\sigma^{a b}{ }_{\mu} \mathrm{d} x^{\mu}$. Note also that $*: \Omega^{p}\left(M_{d}\right) \rightarrow \Omega^{d-p}\left(M_{d}\right)$ denotes the Hodge dual operator.

A usual practice when studying this system's phenomenology is to pack all the torsional terms (coming from the Lorentz curvature (3.7)) to create an extra stress-energy tensor in $(3.11)$.

\section{Conformal Riemann-Cartan structure}

To characterize conformal structures in differential forms language, let us introduce an operator $\mathrm{I}_{a_{1} \ldots a_{q}}: \Omega^{p}\left(M_{d}\right) \rightarrow \Omega^{p-q}\left(M_{d}\right)$ defined in four dimensional spacetime $(-,+,+,+)$ by $[105]$

$$
\mathrm{I}_{a_{1} \ldots a_{q}}=-(-1)^{p(p-q)} * e_{a_{1}} \wedge \ldots \wedge e_{a_{q}} \wedge * .
$$

The case $q=1$ that gives

$$
\mathrm{I}_{a}=-*\left(e_{a} \wedge *\right.
$$

is of particular relevance because it satisfies useful properties. It satisfies the Leibniz rule for differential forms and, together with $\mathrm{D}$, the operator $\mathrm{I}_{a}$ defines another important object $\mathcal{D}_{a}: \Omega^{p}\left(M_{d}\right) \rightarrow \Omega^{p}\left(M_{d}\right)$ via the anti-commutator

$$
\mathcal{D}_{a}=\left\{\mathrm{I}_{a}, \mathrm{D}\right\}=\mathrm{I}_{a} \mathrm{D}+\mathrm{DI}_{a} .
$$

The operators $\mathcal{D}, \mathrm{I}_{a}$ and $\mathrm{D}$, form an open superalgebra where the two-forms curvature and torsion play the role of structure parameters (See [106]).

$$
\begin{aligned}
\left\{\mathrm{I}_{a}, \mathrm{D}\right\} & =\mathcal{D}_{a} \\
\left\{\mathrm{I}_{a}, \mathrm{I}_{b}\right\} & =0 \\
\{\mathrm{D}, \mathrm{D}\} & =2 \mathrm{D}^{2} \\
{\left[\mathrm{I}_{a}, \mathcal{D}_{b}\right] } & =-T_{a b}^{c} \mathrm{I}_{c} \\
{\left[\mathrm{D}, \mathcal{D}_{b}\right] } & =\mathrm{D}^{2} \mathrm{I}_{b}-\mathrm{I}_{b} \mathrm{D}^{2}, \\
{\left[\mathcal{D}_{a}, \mathcal{D}_{b}\right] } & =\mathrm{I}_{a b} \mathrm{D}^{2}+\mathrm{D}^{2} \mathrm{I}_{a b}+\mathrm{I}_{a} \mathrm{D}^{2} \mathrm{I}_{b}-\mathrm{I}_{b} \mathrm{D}^{2} \mathrm{I}_{a}-\left(\mathrm{D} T_{a b}^{c} \wedge \mathrm{I}_{c}+T_{a b}^{c} \mathcal{D}_{c}\right) .
\end{aligned}
$$


The Weyl transformation

$$
g_{\mu \nu}=\exp (2 \varepsilon) \bar{g}_{\mu \nu},
$$

that relates the spacetime and the auxiliary manifolds supposes implicitly that a local mapping $\gamma: M_{4} \rightarrow \bar{M}_{4}$ has been chosen in such a way that the same coordinates $x^{\mu}$ can be used for $P \in M_{4}$ and $\bar{P}=\gamma(P) \in \bar{M}_{4}$. This means that a coordinate transformation $x^{\prime \mu}=$ $x^{\prime \mu}\left(x^{\nu}\right)$ in $M_{4}$ induces the same transformation in $\bar{M}_{4}$ and thus, tensors or forms defined on these manifolds transforms with the same Jacobian matrices. This fact allows us to find the relation between the vielbeins associated with these metrics, which by definition satisfy

$$
\begin{aligned}
& g_{\mu \nu}=e_{\mu}^{a} e_{\nu}^{a} \eta_{a b}, \\
& \bar{g}_{\mu \nu}=\bar{e}_{\mu}^{a} \bar{e}_{\nu}^{b} \eta_{a b} .
\end{aligned}
$$

Indeed, mixing these expressions together with (4.10), it is direct to see that

$$
e^{a}=\exp (\varepsilon) \bar{e}^{a} .
$$

From the vierbein $e^{a}(x)$, it is possible to define "structure parameters" $\mathcal{C}_{a b}^{c}(x)$ satisfying a generalized Maurer-Cartan equation.

$$
\mathrm{d} e^{c}=-\frac{1}{2} \mathcal{C}_{a b}{ }^{c} e^{a} \wedge e^{b} .
$$

This parameter allow us to solve the torsion-free part of the spin connection

$$
\stackrel{\circ}{\omega}_{a b}=\frac{1}{2}\left(\mathcal{C}_{a b c}+\mathcal{C}_{c b a}-\mathcal{C}_{c a b}\right) e^{c} .
$$

Using eqs. (4.13), (4.14) and (4.15), we find

$$
\stackrel{\circ}{\omega}_{a b}=\overline{\dot{\omega}}_{a b}+\bar{e}_{a} \xi_{b}-\xi_{a} \bar{e}_{b},
$$

where

$$
\xi^{a}=\overline{\mathrm{I}}^{a} \mathrm{~d} \varepsilon,
$$

and

$$
\overline{\mathrm{I}}_{a}=-\bar{*}\left(\bar{e}_{a} \wedge \bar{*} .\right.
$$

Note that $\bar{*}$ denotes $\bar{e}^{a}$-vierbein dependence in the Hodge dual operator. In this way, eq. (4.16) characterizes the Weyl transformation associated to the torsion free part of the spin connection.

Notice that we have no information yet on the Weyl transformation of the contorsion $\kappa^{a b}$. This is due to the fact that in the context of Riemann-Cartan geometry, $e^{a}$ and $\kappa^{a b}$ are completely independent degrees of freedom. Therefore, there are multiple possible choices on how $\kappa^{a b}$ should transform under a Weyl transformation. An important family of choices can be parameterized as

$$
\begin{aligned}
\bar{e}^{a} & \rightarrow e^{a}=\exp (\varepsilon) \bar{e}^{a}, \\
\bar{\kappa}_{a b} & \rightarrow \kappa_{a b}=\bar{\kappa}_{a b}+(\lambda-1) \theta_{a b}, \\
\bar{\omega}_{a b} & \rightarrow \omega_{a b}=\bar{\omega}_{a b}+\lambda \theta_{a b},
\end{aligned}
$$


where $\lambda$ is a parameter $0 \leq \lambda \leq 1$ and $\theta^{a b}=-\theta^{b a}$ corresponds to the 1-form

$$
\theta^{a b}=\bar{e}^{a} \xi^{b}-\xi^{a} \bar{e}^{b}
$$

The case $\lambda=1$ implies,

$$
\begin{aligned}
\kappa_{a b} & =\bar{\kappa}_{a b}, \\
\omega_{a b} & =\bar{\omega}_{a b}+\theta_{a b},
\end{aligned}
$$

which is the "canonical case": the full spin connection changes as the torsionless case, and the contorsion is left untouched by the Weyl transformation. The most "exotic" case corresponds to $\lambda=0$

$$
\begin{aligned}
\kappa_{a b} & =\bar{\kappa}_{a b}-\theta_{a b}, \\
\omega_{a b} & =\bar{\omega}_{a b},
\end{aligned}
$$

where the spin connection is left untouched by the Weyl transformation and the contorsion absorbs the transformation.

It is clear that the torsionless condition is preserved only for the $\lambda=1$ case. In fact, the Lorentz curvature and torsion change under the generalized Weyl transformation (4.19)$(4.21)$ as

$$
\begin{aligned}
\bar{T}_{a} & \rightarrow T_{a}=\exp (\varepsilon) \bar{T}_{a}+(\lambda-1) \bar{e}_{a} \wedge \mathrm{d} \exp (\varepsilon), \\
\bar{R}^{a b} & \rightarrow R^{a b}=\bar{R}^{a b}+\lambda \overline{\mathrm{D}} \theta^{a b}+\lambda^{2} \theta^{a}{ }_{c} \wedge \theta^{c b} .
\end{aligned}
$$

\section{$5 \quad$ Mimetic ECSK gravity}

Mimetic transformations are a particular choice of Weyl transformations. Let us consider the auxiliary vierbein $\bar{e}^{a}$ and spin connection $\bar{\omega}^{a b} 1$-forms, and a scalar field $\phi(x)$. In terms of operators $\overline{\mathrm{I}}_{a}$ and $\phi$, let us define a zero-form Lorentz vector

$$
\bar{Z}_{a}=\overline{\mathrm{I}}_{a} \mathrm{~d} \phi,
$$

and the scalar

$$
\bar{Z}^{2}=-\eta_{a b} \bar{Z}^{a} \bar{Z}^{b}
$$

The generalized mimetic vierbein $e^{a}$, contorsion $\kappa^{a b}$, and spin connection $\omega^{a b}$ are defined by

$$
\begin{aligned}
\bar{e}^{a} & \rightarrow e^{a}=\bar{Z} \bar{e}^{a}, \\
\bar{\kappa}_{a b} & \rightarrow \kappa_{a b}=\bar{\kappa}_{a b}+(\lambda-1) \theta_{a b}, \\
\bar{\omega}_{a b} & \rightarrow \omega_{a b}=\bar{\omega}_{a b}+\lambda \theta_{a b},
\end{aligned}
$$

where the one-form $\theta^{a b}$ is given in (4.22) with

$$
\xi^{a}=\frac{1}{\bar{Z}} \overline{\mathrm{I}}^{a} \mathrm{~d} \bar{Z} .
$$


Notice that $\mathrm{I}_{a}$ and $\overline{\mathrm{I}}_{a}$ operator relate each other by

$$
\mathrm{I}_{a}=\frac{1}{\bar{Z}} \overline{\mathrm{I}}_{a}
$$

and consequently

$$
Z_{a}=\frac{1}{\bar{Z}} \bar{Z}_{a}
$$

so the constraint (2.3) reads

$$
Z^{2}=-\eta_{a b} Z^{a} Z^{b}=1
$$

\subsection{Mimetic field equations}

To construct the mimetic version of ECSK theory, let us consider the Lagrangian (3.8), with the vierbein $e^{a}$ and $\omega^{a b}$ in terms of the auxiliary variables $\bar{e}^{a}, \bar{\omega}^{a b}$ and $\phi$ as in eqs. (5.3)(5.5). A priori, it would seem that different choices of $\lambda$ would lead us to different dynamics. In particular, the canonical and exotic choices $\lambda=1$ and $\lambda=0$ seem to lead to completely different theories. However, nothing is further from truth. The dynamics of the generalized mimetic theory is the same regardless of the choice of $\lambda$. Since

$$
\begin{aligned}
e^{a} & =\bar{Z} \bar{e}^{a}, \\
\omega_{a b} & =\bar{\omega}_{a b}+\lambda\left(\bar{e}_{a} \xi_{b}-\xi_{a} \bar{e}_{b}\right),
\end{aligned}
$$

we have that the functional variations of the vierbein and the spin connection are given by

$$
\begin{aligned}
\delta_{\bar{\omega}} e^{d} & =0, \\
\delta_{\bar{e}} e^{d} & =\delta_{\bar{e}} \bar{Z} \bar{e}^{d}+\bar{Z} \delta \bar{e}^{d}, \\
\delta_{\phi} e^{d} & =\delta_{\phi} \bar{Z} \bar{e}^{d},
\end{aligned}
$$

and

$$
\begin{aligned}
\delta_{\bar{\omega}} \omega_{a b} & =\delta \bar{\omega}_{a b}, \\
\delta_{\bar{e}} \omega_{a b} & =\lambda\left(\delta \bar{e}_{a} \xi_{b}-\xi_{a} \delta \bar{e}_{b}\right)+\lambda\left(\bar{e}_{a} \delta_{\bar{e}} \xi_{b}-\delta_{\bar{e}} \xi_{a} \bar{e}_{b}\right), \\
\delta_{\phi} \omega_{a b} & =\lambda\left(\bar{e}_{a} \delta_{\phi} \xi_{b}-\delta_{\phi} \xi_{a} \bar{e}_{b}\right) .
\end{aligned}
$$

Notice that we need special care when performing the functional variation $\delta \bar{Z}^{a}$. In fact, from definition (5.1), it is clear that $\bar{Z}=\bar{Z}(\bar{e}, \partial \phi)$. Therefore, we have to consider independent variations of $\bar{Z}^{a}$ with respect to both, the vierbein $\bar{e}^{a}(x)$ and the scalar field $\phi(x)$. Since

$$
\delta \bar{Z}=-\frac{1}{\bar{Z}} \bar{Z}^{a} \delta \bar{Z}_{a}
$$

it is possible to prove that

$$
\begin{aligned}
& \delta_{\bar{e}} \bar{Z}=\bar{Z}^{2} Z_{a} Z_{b} \mathrm{I}^{a}\left(\delta \bar{e}^{b}\right), \\
& \delta_{\phi} \bar{Z}=-\bar{Z} Z^{a} \mathrm{I}_{a} \mathrm{~d} \delta \phi .
\end{aligned}
$$


Replacing (5.19)-(5.20) into (5.13)-(5.14) we get the expressions

$$
\begin{aligned}
& \delta_{\bar{\omega}} e^{d}=0, \\
& \delta_{\bar{e}} e^{d}=\bar{Z}\left[Z_{a} Z_{b} \mathrm{I}^{a}\left(\delta \bar{e}^{b}\right) e^{d}+\delta \bar{e}^{d}\right], \\
& \delta_{\phi} e^{d}=-e^{d} Z^{a} \mathrm{I}_{a} \mathrm{~d} \delta \phi .
\end{aligned}
$$

Up to boundary terms, variation of (3.8) reads

$$
\begin{aligned}
& \delta_{\bar{e}} \mathcal{L}_{\mathrm{ECSK}}^{(4)}=\frac{1}{\kappa_{4}}\left[\frac{1}{2} \delta_{\bar{e}} \omega^{a b} \wedge \mathcal{W}_{a b}+\mathcal{E}_{d} \wedge \delta_{\bar{e}} e^{d}\right]=0, \\
& \delta_{\phi} \mathcal{L}_{\mathrm{ECSK}}^{(4)}=\frac{1}{\kappa_{4}}\left[\frac{1}{2} \delta_{\phi} \omega^{a b} \wedge \mathcal{W}_{a b}+\mathcal{E}_{d} \wedge \delta_{\phi} e^{d}\right]=0, \\
& \delta_{\bar{\omega}} \mathcal{L}_{\mathrm{ECSK}}=\frac{1}{2} \frac{1}{\kappa_{4}} \delta_{\bar{\omega}} \omega^{a b} \wedge \mathcal{W}_{a b}=0,
\end{aligned}
$$

where the three-forms $\mathcal{E}_{a}$ and $\mathcal{W}_{a b}$ are given in (3.11) and (3.12) respectively. Since $\delta_{\bar{\omega}} \omega_{a b}=$ $\delta \bar{\omega}_{a b}$, eq. (5.26) implies $\delta \bar{\omega}^{a b} \wedge \mathcal{W}_{a b}=0$ and consequently

$$
\mathcal{W}_{a b}=0,
$$

just as in the standard ECSK model. Inserting $\mathcal{W}_{a b}=0$ in the equations of motion, we are left with

$$
\begin{aligned}
& \delta_{\bar{e}} \mathcal{L}_{\mathrm{ECSK}}^{(4)}=\frac{1}{\kappa_{4}} \mathcal{E}_{d} \wedge \delta_{\bar{e}} e^{d}=0, \\
& \delta_{\phi} \mathcal{L}_{\mathrm{ECSK}}^{(4)}=\frac{1}{\kappa_{4}} \mathcal{E}_{d} \wedge \delta_{\phi} e^{d}=0 .
\end{aligned}
$$

From here, using the expressions (5.22) and (5.23), and integrating by parts in $\mathrm{I}^{a}$ and $\mathrm{d}$, we get the set of mimetic ECSK field equations

$$
\begin{aligned}
\mathcal{E}_{d}-Z_{a} Z_{d} \mathrm{I}^{a}\left(\mathcal{E}_{m} \wedge e^{m}\right) & =0, \\
\mathrm{~d}\left[Z^{a} \mathrm{I}_{a}\left(\mathcal{E}_{d} \wedge e^{d}\right)\right] & =0, \\
\mathcal{W}_{a b} & =0 .
\end{aligned}
$$

It is remarkable that they do not depend on the choice of $\lambda$. For the mimetic theory, all the choices of Weyl transformations for the contorsion lead to the same dynamics.

In order to study the equivalence of these equations written using tensors, it is useful to consider Hodge duality between $p$-forms and $(d-p)$-forms. For a three-form $\mathcal{E}_{d}$ in four dimensions, we have

$$
\mathcal{E}_{d}=\mathcal{E}_{m d} * e^{m}
$$

It is straightforward to prove that

$$
\begin{aligned}
\mathcal{E}_{q} \wedge e^{q} & =-\mathcal{E}^{p}{ }_{p} v_{(4)} \\
\mathrm{I}^{m} v_{(4)} & =* e^{m}
\end{aligned}
$$


where $v_{(4)}$ denotes the volume form in four-dimensions. Replacing these relations into the field equations (5.30)-(5.32) it is possible to write them as

$$
\begin{aligned}
\mathcal{E}_{d}-*\left(Z_{d} \mathcal{E}^{p}{ }_{p} \mathrm{~d} \phi\right) & =0, \\
-\mathrm{d} *\left[\mathcal{E}^{p}{ }_{p} \mathrm{~d} \phi\right] & =0, \\
\mathcal{W}_{a b} & =0 .
\end{aligned}
$$

Remarkably, eqs. (5.36)-(5.37) have the same form as eqs. (2.5)-(2.6) but in terms of the full Lorentz curvature (3.7) instead of just the Riemannian piece. Note that eq. (5.38) is the standard field equation for torsion in terms of the spin tensor of matter.

\subsection{Conservation laws}

Both ideas, mimetic gravity and nonvanishing torsion introduce subtle features regarding conservation laws. On the mimetic side, let us observe that eq. (5.36) implies an extra mimetic stress-energy one-form $\overline{\mathcal{T}}_{a}=\overline{\mathcal{T}}_{a b} e^{b}$ corresponding to

$$
\overline{\mathcal{T}}_{d}=\frac{1}{\kappa_{4}} Z_{d} \mathcal{E}^{p}{ }_{p} \mathrm{~d} \phi .
$$

Now, when using the covariant derivative $\mathrm{D}=\mathrm{d}+\omega$, conservation of (5.39) does not hold $\mathrm{D} * \overline{\mathcal{T}}_{d} \neq 0$. In fact,

$$
\begin{aligned}
\mathrm{D} * \overline{\mathcal{T}}_{d} & =\frac{1}{\kappa_{4}} \mathrm{D}\left(Z_{d} *\left[\mathcal{E}^{p}{ }_{p} \mathrm{~d} \phi\right]\right) \\
& =\frac{1}{\kappa_{4}}\left(\mathrm{D} Z_{d} \wedge *\left[\mathcal{E}^{p}{ }_{p} \mathrm{~d} \phi\right]+Z_{d} \wedge \mathrm{D} *\left[\mathcal{E}^{p}{ }_{p} \mathrm{~d} \phi\right]\right) \\
& =\frac{1}{\kappa_{4}}\left(\mathcal{E}^{p}{ }_{p} \mathrm{D} Z_{d} \wedge * \mathrm{~d} \phi+Z_{d} \wedge \mathrm{d} *\left[\mathcal{E}^{p}{ }_{p} \mathrm{~d} \phi\right]\right) .
\end{aligned}
$$

In addition, using eq. (5.37), we have

$$
\begin{aligned}
\mathrm{D} * \overline{\mathcal{T}}_{d} & =\frac{1}{\kappa_{4}} \mathcal{E}^{p}{ }_{p} \mathrm{D} Z_{d} \wedge * \mathrm{~d} \phi \\
& =\frac{1}{\kappa_{4}} \mathcal{E}^{p}{ }_{p} Z^{a} \mathcal{D}_{a} Z_{d} v_{(4)} .
\end{aligned}
$$

Applying (4.7) to $Z_{a}=\mathrm{I}_{a} \mathrm{~d} \phi$, one finds

$$
\mathcal{D}_{a} Z_{d}-\mathcal{D}_{d} Z_{a}=-T^{c}{ }_{a d} Z_{c},
$$

and therefore

$$
\begin{aligned}
\mathrm{D} * \overline{\mathcal{T}}_{d} & =\frac{1}{\kappa_{4}} \mathcal{E}^{p}{ }_{p}\left(Z^{a} \mathcal{D}_{d} Z_{a}-T_{a b d} Z^{a} Z^{b}\right) v_{(4)} \\
& =\frac{1}{\kappa_{4}} \mathcal{E}^{p}{ }_{p}\left(\frac{1}{2} \mathrm{I}_{d} \mathrm{~d}\left(Z^{a} Z_{a}\right)-T_{a b d} Z^{a} Z^{b}\right) v_{(4)} .
\end{aligned}
$$

Finally using eq. (5.9), we arrive to

$$
\begin{aligned}
\mathrm{D} * \overline{\mathcal{T}}_{d} & =-\frac{1}{\kappa_{4}} \mathcal{E}^{p}{ }_{p} T_{a b d} Z^{a} Z^{b} v_{(4)} \\
& =-\overline{\mathcal{T}}^{a b} T_{a b d} v_{(4)} .
\end{aligned}
$$


The result $\mathrm{D} * \overline{\mathcal{T}}_{d} \neq 0$ may seem strange, but it is not an anomaly. This behavior is usual when considering nonvanishing torsion, especially in the context of standard ECSK theory. In order to clarify this point, we must observe that an authentic conservation law for a current one-form $J=J_{\mu} \mathrm{d} x^{\mu}=J_{a} e^{a}$, takes the following form

$$
*(\mathrm{~d} * J)=0 .
$$

Consequently, the generalized Stokes theorem leads us to the integral on the spacetime boundary

$$
\int_{\partial M_{4}} * J=0
$$

It is essential to note that regardless of the torsional background, the conservation relation (5.45) corresponds to

$$
*(\mathrm{~d} * J)=-\mathrm{I}_{a} \stackrel{\circ}{\mathrm{D}} J^{a}=0,
$$

making use of $\mathrm{D}^{\circ}$ and not of D. Thus, in the context of standard ECSK, to write a "conservation law" we use eq. (3.7) to pack all the torsional terms together in the effective torsional stress-energy one form

$$
\mathcal{T}_{d}^{(\mathrm{T})}=-\frac{1}{2 \kappa_{4}} *\left[\left(\epsilon_{a b c d} \stackrel{\circ}{\mathrm{D}} \kappa^{a b}+\epsilon_{a b c d} \kappa^{a}{ }_{e} \wedge \kappa^{e b}\right) \wedge e^{c}\right]
$$

where $\kappa^{a b}$ corresponds to the contorsion one-form. Using (5.48), the ECSK field equation (3.11) is recast as

$$
\frac{1}{2} \epsilon_{a b c d} \stackrel{\circ}{R}^{a b} \wedge e^{c}-\frac{\Lambda}{3 !} \epsilon_{a b c d} e^{a} \wedge e^{b} \wedge e^{c}=\kappa_{4} *\left(\mathcal{T}_{d}+\mathcal{T}_{d}^{(\mathrm{T})}\right) .
$$

This means that a "conservation law" should be written as

$$
\stackrel{\circ}{\mathrm{D}} *\left(\mathcal{T}_{d}+\mathcal{T}_{d}^{(\mathrm{T})}\right)=0,
$$

again making use of $\mathrm{D}^{\mathrm{instead}}$ of D. In conclusion, in the ECSK theory torsion plays the role of a new Riemannian curvature source.

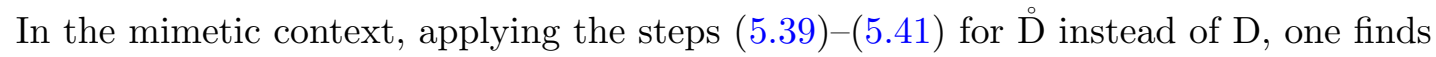

$$
\stackrel{\circ}{\mathrm{D}} * \overline{\mathcal{T}}_{d}=0,
$$

for the effective mimetic stress-energy tensor $\overline{\mathcal{T}}_{d}$, without resorting to an effective torsional stress-energy tensor, in contrast with ECSK theory obeying eq. (5.50). This fact reflects the structure of the mimetic field equations (5.36)-(5.38). In them, only the spin tensor of matter is the torsion source, and there is no torsion- $\phi$ interaction. This lack of interaction leads to eq. (5.51) since $\phi$ and torsion do not interchange energy or momentum.

Of course, it is important to remark that even when a stress-energy one form $\mathcal{T}_{a}=\mathcal{T}_{a b} e^{b}$ obeys $\stackrel{\circ}{D}^{2} \mathcal{T}_{d}=0, \mathcal{T}_{a}$ by itself is not an authentic conserved current one form in the sense of eq. (5.46). Regardless of the torsional background, to construct a real conserved current one form, we must consider $J=\zeta^{a} \mathcal{T}_{a}$, where $\vec{\zeta}=\zeta^{\mu} \partial_{\mu}$ corresponds to a Killing vector. 


\section{The trace of the stress-energy tensor, torsion and $\lambda$}

For the mimetic theory dynamics, the choice of the parameter $\lambda$ for the Weyl transformations (5.3)-(5.5) seems to be irrelevant. However, it does not mean that the parameter is meaningless. In fact, it is related with the value of the trace of stress-energy tensor of matter when its Lagrangian has conformal symmetry.

Let us consider a matter Lagrangian $\mathcal{L}_{\mathrm{M}}$ obeying conformal symmetry by itself

$$
\mathcal{L}_{\mathrm{M}}\left(e^{a}, \omega^{a b}, \varphi\right)=\mathcal{L}_{\mathrm{M}}\left(\Omega e^{a}, \omega^{a b}+\frac{\lambda}{\Omega}\left(e^{a} \mathrm{I}^{b}-e^{b} \mathrm{I}^{a}\right) \mathrm{d} \Omega, \frac{1}{\Omega^{\alpha}} \varphi\right),
$$

where $\Omega=\exp (\varepsilon)$. In the standard torsionless case, it would lead to a traceless on-shell stress-energy tensor. This is no longer true in the current context of non-vanishing torsion. In fact, under an infinitesimal Weyl transformation $\Omega=1+\varepsilon$, the field content of the matter Lagrangian changes according to

$$
\begin{aligned}
& \delta_{\varepsilon} e^{a}=\varepsilon e^{a}, \\
& \delta_{\varepsilon} \omega^{a b}=\lambda \theta^{a b}, \\
& \delta_{\varepsilon} \varphi=-\alpha \varepsilon \varphi,
\end{aligned}
$$

with $\theta^{a b}$ is given in (4.22). Moreover, an arbitrary variation of $\mathcal{L}_{\mathrm{M}}(e, \omega, \varphi)$ reads

$$
\delta \mathcal{L}_{\mathrm{M}}=-* \mathcal{T}_{d} \wedge \delta e^{d}+\frac{1}{2} * \sigma_{a b} \wedge \delta \omega^{a b}+\Phi \delta \varphi+\mathrm{d}\left(\mathcal{B}_{a}^{(2)} \wedge \delta e^{a}+\mathcal{B}_{a b}^{(2)} \wedge \delta \omega^{a b}+\mathcal{B}^{(3)} \delta \varphi\right),
$$

where $\Phi$ denotes the variation of $\mathcal{L}_{\mathrm{M}}$ with respect to $\varphi$ and $\mathcal{B}_{a}, \mathcal{B}_{a b}$, and $\mathcal{B}$ are boundary terms. Therefore, demanding invariance of $\mathcal{L}_{\mathrm{M}}$ under the infinitesimal Weyl transformations (6.2)-(6.4) we obtain

$$
\varepsilon e^{d} \wedge * \mathcal{T}_{d}+\lambda * \sigma_{a b} \wedge e^{a} \xi^{b}-\alpha \varepsilon \Phi \varphi+\mathrm{d}\left(\mathcal{B}_{a}^{(2)} \wedge \varepsilon e^{a}+2 \lambda \mathcal{B}_{a b}^{(2)} \wedge e^{a} \xi^{b}-\alpha \varepsilon \mathcal{B}^{(3)} \varphi\right)=0 .
$$

It is straightforward to prove the identity

$$
* \sigma_{a b} \wedge e^{a} \xi^{b}=-\mathrm{d}\left[\varepsilon \mathrm{I}^{b}\left(e^{a} \wedge * \sigma_{a b}\right)\right]+\varepsilon \mathrm{dI}^{b}\left(e^{a} \wedge * \sigma_{a b}\right),
$$

and therefore, integrating (6.6), to conclude that

$$
\int_{M_{4}} \mathcal{H}^{(4)}+\int_{\partial M_{4}} \mathcal{U}^{(3)}=0
$$

where

$$
\mathcal{H}^{(4)}=\varepsilon\left[e^{d} \wedge * \mathcal{T}_{d}+\lambda \mathrm{dI}^{b}\left(e^{a} \wedge * \sigma_{a b}\right)-\alpha \Phi \varphi\right]
$$

and

$$
\mathcal{U}^{(3)}=\varepsilon \mathcal{B}_{a}^{(2)} \wedge e^{a}+\lambda\left[2 \mathcal{B}_{a b}^{(2)} \wedge e^{a} \xi^{b}-\varepsilon \mathrm{I}^{b}\left(e^{a} \wedge * \sigma_{a b}\right)\right]-\alpha \varepsilon \mathcal{B}^{(3)} \varphi .
$$

Since $\varepsilon$ is local and arbitrary parameter, the integrals over the bulk and boundary must vanish independently. Neglecting boundary contributions, from (6.9) we are left with

$$
e^{d} \wedge * \mathcal{T}_{d}+\lambda \mathrm{dI}^{b}\left(e^{a} \wedge * \sigma_{a b}\right)-\alpha \Phi \varphi=0 .
$$


Note that in differential forms language the trace of the stress-energy tensor is written as $\mathcal{T}=-*\left(e^{d} \wedge * \mathcal{T}_{d}\right)$. In this way, taking the Hodge dual in (6.11) we find

$$
\mathcal{T}-\lambda * \mathrm{dI}^{b}\left(e^{a} \wedge * \sigma_{a b}\right)+\alpha * \Phi \varphi=0 .
$$

Using (4.2) it is possible to probe that $\mathrm{I}^{b}\left(e^{a} \wedge * \sigma_{a b}\right)=* e^{b} \sigma^{a}{ }_{a b}$. Furthermore, in a theory as ECSK or it current mimetic version, imposing the on-shell condition implies for one hand $\Phi=0$, and on the other hand, equation (3.12) allows to solve the components of the spin tensor in terms of the components of torsion. In fact

$$
\sigma_{b a}^{a}=\frac{2}{\kappa_{4}} T_{a b}^{a},
$$

and replacing (6.13) in eq. (6.12) on-shell, it lead us to the trace value

$$
\mathcal{T}=\frac{2 \lambda}{\kappa_{4}} \mathrm{~d}^{\dagger} \mathrm{I}_{a} T^{a}
$$

with the four-dimensional coderivative operator $\mathrm{d}^{\dagger}$ given by $\mathrm{d}^{\dagger}=* \mathrm{~d} *$. This way, we can see that the stress-energy tensor for a Weyl transformation invariant Lagrangian is not always traceless. It is traceless only when the torsion vanishes or when the Weyl transformation invariance is associated to the case $\lambda=0$, i.e. when the spin connection remains untouched by the transformation.

\section{Summary \& comments}

In summary, we have developed the closest version of mimetic gravity in first-order formalism, i.e., the mimetic version of ECSK gravity theory. Dynamics in this theory is described by the following equations of motion

$$
\begin{aligned}
\mathcal{E}_{d}-\kappa_{4} * \overline{\mathcal{T}}_{d} & =0, \\
\mathrm{~d} *\left[\mathcal{E}^{p}{ }_{p} \mathrm{~d} \phi\right] & =0, \\
\mathcal{W}_{a b} & =0,
\end{aligned}
$$

where $\mathcal{E}_{a}, \mathcal{W}_{a b}$, and $\overline{\mathcal{T}}_{d}$ are given in eqs. (3.11), (3.12), and (5.39) respectively. By construction, the system obeys the following conditions:

$$
\begin{aligned}
\stackrel{\circ}{\mathrm{D} * \overline{\mathcal{T}}_{d}} & =0, \\
Z^{2} & =1 .
\end{aligned}
$$

These equations reduce to the standard mimetic gravity equation when torsion $T^{a}$ vanishes.

We have considered different possibilities of how torsion is affected by Weyl transformation (2.1), all of them mapped by the parameter $\lambda$. The torsionless part of the spin connection $\stackrel{\llcorner}{\omega}^{a b}$ has a definite Weyl transformation (4.15), obtained from purely metric properties. However, we have the freedom to choose the contorsion $\kappa^{a b}$ transformation. The possibilities (4.19)-(4.21) range from a non-changing contorsion, to a contorsion that 
changes in such a way that it leaves the full spin connection invariant. Regardless of the type of transformation under consideration, dynamics enforce torsion to remain a nonpropagating field, as in standard ECSK.

An interesting application of this model is in the context of cosmology. ${ }^{2}$ Let us consider an explicit solution of equation (7.2). For the sake of simplicity, during this section we take $c=1$ and $\Lambda=0$. Additionally, it is convenient to work in synchronous coordinates where the metric adopts the form

$$
\mathrm{d} s^{2}=-\mathrm{d} \tau^{2}+\gamma_{i j} \mathrm{~d} x^{i} \mathrm{~d} x^{j},
$$

with $\gamma_{i j}$ the spatial section of the metric $g_{\mu \nu}$ (See [138, Chap.11]). As discussed in [41], we take the scalar field to be the same as the hypersurfaces of constant time, namely

$$
\phi\left(x^{\mu}\right)=\tau,
$$

which naturally satisfies (7.5). In these coordinates, eq. (7.2) reads

$$
\partial_{0}(\sqrt{\gamma}(G-\mathcal{T}))=0,
$$

and consequently

$$
G-\mathcal{T}=\frac{\mathcal{C}\left(x^{i}\right)}{\sqrt{\gamma}}
$$

Here $\mathcal{C}$ is an integration $\tau$-constant, depending only on the spatial coordinates $x^{i}$. For a flat Friedmann Universe, the metric $\gamma_{i j}$ corresponds to

$$
\gamma_{i j}=a^{2}(\tau) \delta_{i j}
$$

and therefore (7.8) leads to

$$
G-\mathcal{T}=\frac{\mathcal{C}\left(x^{i}\right)}{a^{3}(\tau)} .
$$

Therefore, the scalar field coming from the conformal degree of freedom mimics a dark matter source. However, torsion is also present, and it behaves as an additional dark matter source in $G=-R$. Let us split the Ricci scalar as

$$
\begin{aligned}
R & =\stackrel{\circ}{R}+R(\kappa), \\
R(\kappa) & =2 \stackrel{\circ}{\nabla}_{\mu} \kappa^{\mu \nu}{ }_{\nu}+\kappa^{\mu \gamma}{ }_{\mu} \kappa_{\gamma \nu}{ }^{\nu}-\kappa^{\mu}{ }_{\gamma \nu} \kappa^{\gamma \nu}{ }_{\mu},
\end{aligned}
$$

where $\kappa^{\mu \nu}{ }_{\lambda}$ is the contortion, $\stackrel{\circ}{R}$ is the torsionless Ricci scalar and $\stackrel{\circ}{\nabla}^{\circ}$ is the covariant derivative associated with the Christoffel symbol. Thus, using $\stackrel{\circ}{G}=-\stackrel{\circ}{R}$, we get

$$
G=\stackrel{\circ}{G}-R(\kappa) .
$$

In order to evaluate eq. (7.13) explicitly, let us consider a spin tensor distribution $\sigma_{a b}$ which may be relevant at cosmological scales. Such spin tensor distribution has been considered, for instance, in [40] where the Ansatz for the torsion tensor reads

$$
T_{\lambda \mu \nu}=\left[X(\tau)\left(g_{\lambda \nu} g_{\mu \rho}-g_{\lambda \mu} g_{\nu \rho}\right)-2 \sqrt{g} Y(\tau) \epsilon_{\lambda \mu \nu \rho}\right] u^{\rho},
$$

\footnotetext{
${ }^{2}$ By the way, almost simultaneous with this article, another interesting cosmological application of mimetic theories of gravity involving non-vanishing torsion has been presented in [137].
} 
where $u^{\rho}=\delta_{0}^{\rho}$ in synchronous coordinates, and $X$ and $Y$ are arbitrary functions of time. These configurations can arise from a dark matter candidates, such as dark/Elko spinors, see for instance [139-145]. The non-vanishing components of (7.15) are

$$
\begin{aligned}
& T_{i j 0}=-T_{i 0 j}=X \gamma_{i j} u^{0}=a^{2} X \delta_{i j}, \\
& T_{k i j}=-2 \sqrt{\gamma} Y \epsilon_{k i j} u^{0}=-2 a^{3} Y \epsilon_{k i j} .
\end{aligned}
$$

Since

$$
\kappa_{\mu \nu \lambda}=\frac{1}{2}\left(T_{\nu \mu \lambda}-T_{\mu \nu \lambda}+T_{\lambda \mu \nu}\right)
$$

we can evaluate eq. (7.13) and eqs. (7.11)-(7.14) to arrive to

$$
\stackrel{\circ}{G}-\mathcal{T}=\frac{\mathcal{C}}{a^{3}}+3 !\left[X(\mathcal{S}+3 \mathcal{H})+X^{2}+Y^{2}\right],
$$

where $\mathcal{C}=\mathcal{C}\left(x^{i}\right), \mathcal{S}(\tau)=\frac{\dot{X}}{X}$ and $\mathcal{H}(\tau)=\frac{\dot{a}}{a}$. From here the role of the spin-tensor as dark matter becomes evident.

It is interesting to observe that in mimetic ECSK theory, there are three dark matter species. The first is the dark matter by itself, with a non-vanishing spin tensor. The second arises from isolating the conformal mode in a covariant way, and the last comes from the torsional degrees of freedom. In particular, from eq. (7.19) one sees that dark matter arises as a direct sum of contributions coming form mimetic dark matter and torsion separately. This is no longer the case when consider more elaborated mimetic theories containing nonminimal couplings of scalar fields with geometry (See [105]). In such a scenario, a complex interplay between $\phi$ and propagating torsion creates dark matter effects.

This behavior is due to a general result. In ref. [105], we proved that for the Horndeski Lagrangian on a Riemann-Cartan geometry, the nonminimal couplings with the scalar field and second-order terms in the Lagrangian (e.g, a $\square \phi$ term) are generic sources of torsion. The same will happen in the mimetic context. Observing how the structure of the mimetic equations of motion came to be (see, for instance, eqs. (5.24)-(5.26)), it is clear that the mimetic mapping to a new theory preserves the propagating/non-propagating torsion character of the original theory. In both cases, propagating and non-propagating, nonvanishing torsion influences cosmic evolution. In a non-propagating context, torsion and $\phi$ do not interchange energy during the cosmic evolution, as we can see from the former analysis. However, when considering theories with nonminimal couplings or second-order terms in the Lagrangian, in general, $\phi$ and torsion's evolution get completely interrelated; see, for instance, refs. [36, 37, 112, 114].

The non-propagating torsion's simplicity may give the false impression of it being uninteresting in a cosmological setting. That would be far from the truth; for instance, a non-propagating torsion can straightforwardly explain the Hubble parameter tension [40].

\section{Acknowledgments}

We are grateful to Cristian Martínez, Cristóbal Corral, Subhasish Chakrabarty, Amitabha Lahiri, Alfredo Pérez, and Adolfo Cisterna for many enlightening conversations and references. P. M. is supported by Agencia Nacional de Investigación (ANID) through the 
grant 21161574. OV acknowledges VRIIP UNAP for financial support through Project VRIIP0062-19 and Becas Chile grant 74200062. FI ad PS acknowledges financial support from the Chilean government through Fondecyt grant 1180681. NM, acknowledges Fondecyt grant 11180894.

Open Access. This article is distributed under the terms of the Creative Commons Attribution License (CC-BY 4.0), which permits any use, distribution and reproduction in any medium, provided the original author(s) and source are credited.

\section{References}

[1] C.M. Will, The confrontation between general relativity and experiment, Living Rev. Rel. 17 (2014) 4 [arXiv: 1403.7377] [INSPIRE].

[2] Event Horizon Telescope collaboration, First M87 Event Horizon Telescope results. I. The shadow of the supermassive black hole, Astrophys. J. 875 (2019) L1 [arXiv: 1906.11238] [INSPIRE].

[3] LIGO Scientific, VIRGO collaboration, GW170817: observation of gravitational waves from a binary neutron star inspiral, Phys. Rev. Lett. 119 (2017) 161101 [arXiv:1710.05832] [INSPIRE].

[4] Ligo Scientific, Virgo, Fermi-GBM, INTEGRAL collaboration, Gravitational waves and gamma-rays from a binary neutron star merger: GW170817 and GRB 170817A, Astrophys. J. Lett. 848 (2017) L13 [arXiv:1710.05834] [INSPIRE].

[5] LIGO SCIENTIFIC et al. collaborations, Multi-messenger observations of a binary neutron star merger, Astrophys. J. Lett. 848 (2017) L12 [arXiv:1710.05833] [INSPIRE].

[6] Planck collaboration, Planck 2018 results. X. Constraints on inflation, Astron. Astrophys. 641 (2020) A10 [arXiv:1807.06211] [INSPIRE].

[7] G.B. Gelmini, The hunt for dark matter, in the proceedings of the Theoretical Advanced Study Institute in Elementary Particle Physics (TASI 2015), June 1-26, Boulder, U.S.A. (2015), arXiv: 1502.01320 [INSPIRE].

[8] G. Bertone, D. Hooper and J. Silk, Particle dark matter: evidence, candidates and constraints, Phys. Rept. 405 (2005) 279 [hep-ph/0404175] [INSPIRE].

[9] B.L. Young, A survey of dark matter and related topics in cosmology, Front. Phys. 12 (2016) 121201.

[10] P. Bull et al., Beyond $\Lambda$ CDM: problems, solutions, and the road ahead, Phys. Dark Univ. 12 (2016) 56 [arXiv:1512.05356] [INSPIRE].

[11] A. De Felice and S. Tsujikawa, $f(R)$ theories, Living Rev. Rel. 13 (2010) 3 [arXiv: 1002.4928] [INSPIRE].

[12] G.J. Olmo, Palatini approach to modified gravity: $f(R)$ theories and beyond, Int. J. Mod. Phys. D 20 (2011) 413 [arXiv:1101.3864] [inSPIRE].

[13] S. Capozziello and M. De Laurentis, Extended theories of gravity, Phys. Rept. 509 (2011) 167 [arXiv: 1108.6266] [INSPIRE]. 
[14] S. Nojiri, S.D. Odintsov and V.K. Oikonomou, Modified gravity theories on a nutshell: inflation, bounce and late-time evolution, Phys. Rept. 692 (2017) 1 [arXiv:1705.11098] [INSPIRE].

[15] J. Beltran Jimenez, L. Heisenberg, G.J. Olmo and D. Rubiera-Garcia, Born-Infeld inspired modifications of gravity, Phys. Rept. 727 (2018) 1 [arXiv:1704.03351] [INSPIRE].

[16] L. Heisenberg, A systematic approach to generalisations of general relativity and their cosmological implications, Phys. Rept. 796 (2019) 1 [arXiv:1807.01725] [INSPIRE].

[17] F.W. Hehl, P. Von Der Heyde, G.D. Kerlick and J.M. Nester, General relativity with spin and torsion: foundations and prospects, Rev. Mod. Phys. 48 (1976) 393 [InSPIRE].

[18] F.W. Hehl, J. McCrea, E.W. Mielke and Y. Ne'eman, Metric affine gauge theory of gravity: field equations, Noether identities, world spinors, and breaking of dilation invariance, Phys. Rept. 258 (1995) 1 [gr-qc/9402012] [INSPIRE].

[19] A. Trautman, Einstein-Cartan theory, gr-qc/0606062 [INSPIRE].

[20] F.W. Hehl, Y.N. Obukhov and D. Puetzfeld, On Poincaré gauge theory of gravity, its equations of motion, and gravity probe B, Phys. Lett. A 377 (2013) 1775 [arXiv:1304.2769] [INSPIRE].

[21] M. Blagojević and F.W. Hehl, Gauge theories of gravitation, World Scientific, Singapore (2013).

[22] H.I. Arcos and J.G. Pereira, Torsion gravity: a reappraisal, Int. J. Mod. Phys. D 13 (2004) 2193 [gr-qc/0501017] [INSPIRE].

[23] N.J. Poplawski, Nonsingular, big-bounce cosmology from spinor-torsion coupling, Phys. Rev. D 85 (2012) 107502 [arXiv: 1111.4595] [INSPIRE].

[24] G. Unger and N. Popławski, Big bounce and closed universe from spin and torsion, Astrophys. J. 870 (2019) 78 [arXiv: 1808.08327] [INSPIRE].

[25] D. Kranas, C.G. Tsagas, J.D. Barrow and D. Iosifidis, Friedmann-like universes with torsion, Eur. Phys. J. C 79 (2019) 341 [arXiv:1809.10064] [InSPIRE].

[26] N.J. Popławski, Cosmology with torsion: an alternative to cosmic inflation, Phys. Lett. B 694 (2010) 181 [Erratum ibid. 701 (2011) 672] [arXiv:1007.0587] [INSPIRE].

[27] A.N. Ivanov and M. Wellenzohn, Einstein-Cartan gravity with torsion field serving as an origin for the cosmological constant or dark energy density, Astrophys. J. 829 (2016) 47 [arXiv: 1607.01128] [INSPIRE].

[28] O. Razina, Y. Myrzakulov, N. Serikbayev, G. Nugmanova and R. Myrzakulov, Einstein-Cartan gravity with scalar-fermion interactions, Central Eur. J. Phys. 10 (2012) 47 [arXiv: 1012.5690] [INSPIRE].

[29] D. Palle, On the Einstein-Cartan cosmology vs. Planck data, J. Exp. Theor. Phys. 118 (2014) 587 [arXiv:1405.3435] [INSPIRE].

[30] N.J. Poplawski, Thermal fluctuations in Einstein-Cartan-Sciama-Kibble-Dirac bouncing cosmology, arXiv:1201.0316 [INSPIRE].

[31] D.Z. Freedman and A.V. Proeyen, Supergravity, Cambridge University Press, Cambridge U.K. (2012).

[32] D. Puetzfeld and Y.N. Obukhov, Prospects of detecting spacetime torsion, Int. J. Mod. Phys. D 23 (2014) 1442004 [arXiv:1405.4137] [INSPIRE]. 
[33] S.M. Carroll and G.B. Field, Consequences of propagating torsion in connection dynamic theories of gravity, Phys. Rev. D 50 (1994) 3867 [gr-qc/9403058] [InSPIRE].

[34] J. Boos and F.W. Hehl, Gravity-induced four-fermion contact interaction implies gravitational intermediate $W$ and $Z$ type gauge bosons, Int. J. Theor. Phys. 56 (2017) 751 [arXiv: 1606.09273] [INSPIRE].

[35] A. Tilquin and T. Schucker, Torsion, an alternative to dark matter?, Gen. Rel. Grav. 43 (2011) 2965 [arXiv: 1104.0160] [INSPIRE].

[36] S. Alexander et al., Cosmology of minimal varying Lambda theories, Phys. Rev. D 100 (2019) 083507 [arXiv: 1905.10382] [inSPIRE].

[37] J.a. Magueijo and T. Złośnik, Parity violating Friedmann Universes, Phys. Rev. D 100 (2019) 084036 [arXiv: 1908.05184] [INSPIRE].

[38] W.E.V. Barker, A.N. Lasenby, M.P. Hobson and W.J. Handley, Systematic study of background cosmology in unitary Poincaré gauge theories with application to emergent dark radiation and $H_{0}$ tension, Phys. Rev. D 102 (2020) 024048 [arXiv: 2003.02690] [InSPIRE].

[39] S. Alexander, L. Jenks, P. Jiroušek, J.a. Magueijo and T. Złośnik, Gravity waves in parity-violating Copernican Universes, Phys. Rev. D 102 (2020) 044039 [arXiv: 2001.06373] [INSPIRE].

[40] F. Izaurieta, S. Lepe and O. Valdivia, The spin tensor of dark matter and the Hubble parameter tension, Phys. Dark Univ. 30 (2020) 100662 [arXiv: 2004.13163] [INSPIRE].

[41] A.H. Chamseddine and V. Mukhanov, Mimetic dark matter, JHEP 11 (2013) 135 [arXiv:1308.5410] [INSPIRE].

[42] A.H. Chamseddine, V. Mukhanov and A. Vikman, Cosmology with mimetic matter, JCAP 06 (2014) 017 [arXiv: 1403.3961] [INSPIRE].

[43] D. Momeni, R. Myrzakulov and E. Güdekli, Cosmological viable mimetic $f(R)$ and $f(R, T)$ theories via Noether symmetry, Int. J. Geom. Meth. Mod. Phys. 12 (2015) 1550101 [arXiv: 1502.00977] [INSPIRE].

[44] M.A. Gorji, A. Allahyari, M. Khodadi and H. Firouzjahi, Mimetic black holes, Phys. Rev. D 101 (2020) 124060 [arXiv: 1912.04636] [INSPIRE].

[45] A.H. Chamseddine, V. Mukhanov and T.B. Russ, Non-flat universes and black holes in asymptotically free mimetic gravity, Fortsch. Phys. 68 (2020) 1900103 [arXiv:1912.03162] [INSPIRE].

[46] A. Sheykhi and S. Grunau, Topological black holes in mimetic gravity, arXiv:1911.13072 [INSPIRE].

[47] A.H. Chamseddine, V. Mukhanov and T.B. Russ, Black hole remnants, JHEP 10 (2019) 104 [arXiv: 1908.03498] [INSPIRE].

[48] A. Sheykhi, Thermodynamics of apparent horizon in mimetic cosmology, Int. J. Mod. Phys. D 28 (2018) 1950057 [INSPIRE].

[49] G. Nashed, Charged and non-charged black hole solutions in mimetic gravitational theory, Symmetry 10 (2018) 559 [INSPIRE].

[50] G.G.L. Nashed, W. El Hanafy and K. Bamba, Charged rotating black holes coupled with nonlinear electrodynamics Maxwell field in the mimetic gravity, JCAP 01 (2019) 058 [arXiv: 1809.02289] [INSPIRE]. 
[51] G.G.L. Nashed, Spherically symmetric black hole solution in mimetic gravity and anti-evaporation, Int. J. Geom. Meth. Mod. Phys. 15 (2018) 1850154 [INSPIRE].

[52] C.-Y. Chen, M. Bouhmadi-López and P. Chen, The mimetic born-infeld gravity: the primordial cosmos and spherically symmetric solutions, Galaxies 5 (2017) 87 [INSPIRE].

[53] V.K. Oikonomou, A note on Schwarzschild-de Sitter black holes in mimetic $F(R)$ gravity, Int. J. Mod. Phys. D 25 (2016) 1650078 [arXiv:1605.00583] [InSPIRE].

[54] V.K. Oikonomou, Reissner-Nordström Anti-de Sitter black holes in mimetic $F(R)$ gravity, Universe 2 (2016) 10 [arXiv: 1511.09117] [INSPIRE].

[55] A. Sheykhi, Mimetic black strings, JHEP 07 (2020) 031 [arXiv:2002.11718] [INSPIRE].

[56] K. Nozari and N. Sadeghnezhad, Braneworld mimetic $f(R)$ gravity, Int. J. Geom. Meth. Mod. Phys. 16 (2019) 1950042 [inSPIRE].

[57] S. Davood Sadatian and A. Sepehri, Tachyonic braneworld mimetic cosmology, Mod. Phys. Lett. A 34 (2019) 1950162 [INSPIRE].

[58] T.-T. Sui, Y.-P. Zhang, B.-M. Gu and Y.-X. Liu, Different asymptotic behaviors of thick branes in mimetic gravity, arXiv:2005.08438 [INSPIRE].

[59] W.-D. Guo, Y. Zhong, K. Yang, T.-T. Sui and Y.-X. Liu, Thick brane in mimetic $f(T)$ gravity, Phys. Lett. B 800 (2020) 135099 [arXiv:1805.05650] [INSPIRE].

[60] Y. Zhong, Y. Zhong, Y.-P. Zhang and Y.-X. Liu, Thick branes with inner structure in mimetic gravity, Eur. Phys. J. C 78 (2018) 45 [arXiv:1711.09413] [InSPIRE].

[61] N. Sadeghnezhad and K. Nozari, Braneworld mimetic cosmology, Phys. Lett. B 769 (2017) 134 [arXiv: 1703.06269] [INSPIRE].

[62] O. Malaeb and C. Saghir, Mimetic Hořava gravity and surface terms, arXiv: 2005.02469 [INSPIRE].

[63] A. Casalino, L. Sebastiani, L. Vanzo and S. Zerbini, Higher derivative and mimetic models on non flat FLRW space-times, Phys. Dark Univ. 29 (2020) 100594 [arXiv:1912.09307] [INSPIRE].

[64] K. Nozari and N. Rashidi, Mimetic DBI inflation in confrontation with Planck2018 data, Astrophys. J. 882 (2019) 78 [arXiv:1912.06050] [INSPIRE].

[65] L. Shen, Y. Zheng and M. Li, Two-field mimetic gravity revisited and Hamiltonian analysis, JCAP 12 (2019) 026 [arXiv: 1909.01248] [INSPIRE].

[66] H. Ramo Chothe, A. Dutta and S. Sur, Cosmological dark sector from a mimetic-metric-torsion perspective, Int. J. Mod. Phys. D 28 (2019) 1950174 [arXiv: 1907.12429] [INSPIRE].

[67] A. Ganz, N. Bartolo and S. Matarrese, Towards a viable effective field theory of mimetic gravity, JCAP 12 (2019) 037 [arXiv:1907.10301] [INSPIRE].

[68] A.R. Khalifeh, N. Bellomo, J.L. Bernal and R. Jimenez, Can dark matter be geometry? A case study with mimetic dark matter, Phys. Dark Univ. 30 (2020) 100646 [arXiv: 1907.03660] [INSPIRE].

[69] E. Bezerra and O.D. Miranda, Mimetic gravity: mimicking the dynamics of the primeval universe in the context of loop quantum cosmology, Eur. Phys. J. C 79 (2019) 310 [arXiv: 1904.04883] [INSPIRE]. 
[70] M. de Cesare, Reconstruction of mimetic gravity in a non-singularbouncing universe from quantum gravity, Universe 5 (2019) 107 [arXiv: 1904.02622] [INSPIRE].

[71] A.R. Solomon, V. Vardanyan and Y. Akrami, Massive mimetic cosmology, Phys. Lett. B 794 (2019) 135 [arXiv: 1902.08533] [INSPIRE].

[72] O. Malaeb and C. Saghir, Hamiltonian formulation of ghost free mimetic massive gravity, Eur. Phys. J. C 79 (2019) 584 [arXiv:1901.06727] [INSPIRE].

[73] Y. Zhong, Y.-P. Zhang, W.-D. Guo and Y.-X. Liu, Gravitational resonances in mimetic thick branes, JHEP 04 (2019) 154 [arXiv: 1812.06453] [INSPIRE].

[74] M. de Cesare, Limiting curvature mimetic gravity for group field theory condensates, Phys. Rev. D 99 (2019) 063505 [arXiv: 1812.06171] [INSPIRE].

[75] A. Casalino, M. Rinaldi, L. Sebastiani and S. Vagnozzi, Alive and well: mimetic gravity and a higher-order extension in light of GW170817, Class. Quant. Grav. 36 (2019) 017001 [arXiv: 1811.06830] [INSPIRE].

[76] A. Ganz, N. Bartolo, P. Karmakar and S. Matarrese, Gravity in mimetic scalar-tensor theories after GW170817, JCAP 01 (2019) 056 [arXiv: 1809.03496] [INSPIRE].

[77] X.-z. Li, X.-h. Zhai and P. Li, Generalized Birkhoff theorem and its applications in mimetic gravity, arXiv:1807.08270 [INSPIRE].

[78] M.A. Gorji, S. Mukohyama, H. Firouzjahi and S.A. Hosseini Mansoori, Gauge field mimetic cosmology, JCAP 08 (2018) 047 [arXiv: 1807.06335] [inSPIRE].

[79] S.A. Paston and A.A. Sheykin, Embedding theory as new geometrical mimetic gravity, Eur. Phys. J. C 78 (2018) 989 [arXiv:1806.10902] [InSPIRE].

[80] N. Bodendorfer, F.M. Mele and J. Münch, Is limiting curvature mimetic gravity an effective polymer quantum gravity?, Class. Quant. Grav. 35 (2018) 225001 [arXiv:1806.02052] [INSPIRE].

[81] A.H. Chamseddine and V. Mukhanov, Mimetic massive gravity: beyond linear approximation, JHEP 06 (2018) 062 [arXiv:1805.06598] [INSPIRE].

[82] A.H. Chamseddine and V. Mukhanov, Ghost free mimetic massive gravity, JHEP 06 (2018) 060 [arXiv: 1805. 06283] [INSPIRE].

[83] E. Babichev, D. Gorbunov and S. Ramazanov, Dark matter and baryon asymmetry from the very dawn of the Universe, Phys. Rev. D 97 (2018) 123543 [arXiv:1805.05904] [inSPIRE].

[84] Y. Zhong and D. Sáez-Chillón Gómez, Inflation in mimetic $f(G)$ gravity, Symmetry 10 (2018) 170 [arXiv: 1805.03467] [INSPIRE].

[85] S. Brahma, A. Golovnev and D.-H. Yeom, On singularity-resolution in mimetic gravity, Phys. Lett. B 782 (2018) 280 [arXiv: 1803.03955] [INSPIRE].

[86] A. Casalino, M. Rinaldi, L. Sebastiani and S. Vagnozzi, Mimicking dark matter and dark energy in a mimetic model compatible with GW170817, Phys. Dark Univ. 22 (2018) 108 [arXiv: 1803.02620] [inSPIRE].

[87] D. Langlois, M. Mancarella, K. Noui and F. Vernizzi, Mimetic gravity as DHOST theories, JCAP 02 (2019) 036 [arXiv: 1802.03394] [INSPIRE].

[88] S.D. Odintsov and V.K. Oikonomou, The reconstruction of $f(\phi) R$ and mimetic gravity from viable slow-roll inflation, Nucl. Phys. B 929 (2018) 79 [arXiv:1801.10529] [InSPIRE]. 
[89] A. Golovnev, Beyond dRGT as mimetic massive gravity, Phys. Lett. B 779 (2018) 441 [arXiv: 1801.07958] [INSPIRE].

[90] B. Mirza and F. Oboudiat, Mimetic $f(T)$ teleparallel gravity and cosmology, Gen. Rel. Grav. 51 (2019) 96 [arXiv:1712.03363] [INSPIRE].

[91] Z. Haghani and S. Shahidi, Late-time acceleration via mimetic Galileon gravity, in the proceedings of the $14^{\text {th }}$ Marcel Grossmann Meeting, June 17-21, Rome, Italy (2017).

[92] J. Dutta, W. Khyllep, E.N. Saridakis, N. Tamanini and S. Vagnozzi, Cosmological dynamics of mimetic gravity, JCAP 02 (2018) 041 [arXiv:1711.07290] [INSPIRE].

[93] S. Nojiri, S.D. Odintsov and V.K. Oikonomou, Ghost-free $F(R)$ gravity with Lagrange multiplier constraint, Phys. Lett. B $\mathbf{7 7 5}$ (2017) 44 [arXiv:1710.07838] [INSPIRE].

[94] M.A. Gorji, S.A. Hosseini Mansoori and H. Firouzjahi, Higher derivative mimetic gravity, JCAP 01 (2018) 020 [arXiv: 1709. 09988] [INSPIRE].

[95] G.L. Volkmer and D. Hadjimichef, Mimetic dark matter in pseudo-complex general relativity, Int. J. Mod. Phys. Conf. Ser. 45 (2017) 1760012 [InSPIRE].

[96] S.A. Paston, Forms of action for perfect fluid in general relativity and mimetic gravity, Phys. Rev. D 96 (2017) 084059 [arXiv: 1708.03944] [INSPIRE].

[97] F. Arroja, T. Okumura, N. Bartolo, P. Karmakar and S. Matarrese, Large-scale structure in mimetic Horndeski gravity, JCAP 05 (2018) 050 [arXiv:1708.01850] [INSPIRE].

[98] S. Vagnozzi, Recovering a MOND-like acceleration law in mimetic gravity, Class. Quant. Grav. 34 (2017) 185006 [arXiv: 1708.00603] [INSPIRE].

[99] E.H. Baffou, M.J.S. Houndjo, M. Hamani-Daouda and F.G. Alvarenga, Late time cosmological approach in mimetic $f(R, T)$ gravity, Eur. Phys. J. C 77 (2017) 708 [arXiv:1706.08842] [INSPIRE].

[100] L. Sebastiani, S. Vagnozzi and R. Myrzakulov, Mimetic gravity: a review of recent developments and applications to cosmology and astrophysics, Adv. High Energy Phys. 2017 (2017) 3156915 [arXiv:1612.08661] [INSPIRE].

[101] G. Cognola, R. Myrzakulov, L. Sebastiani, S. Vagnozzi and S. Zerbini, Covariant Hořava-like and mimetic Horndeski gravity: cosmological solutions and perturbations, Class. Quant. Grav. 33 (2016) 225014 [arXiv:1601.00102] [INSPIRE].

[102] R. Myrzakulov, L. Sebastiani, S. Vagnozzi and S. Zerbini, Static spherically symmetric solutions in mimetic gravity: rotation curves and wormholes, Class. Quant. Grav. 33 (2016) 125005 [arXiv: 1510.02284] [INSPIRE].

[103] N. Deruelle and J. Rua, Disformal transformations, veiled general relativity and mimetic gravity, JCAP 09 (2014) 002 [arXiv: 1407.0825] [INSPIRE].

[104] S. Chakrabarty and A. Lahiri, Different types of torsion and their effect on the dynamics of fields, Eur. Phys. J. Plus 133 (2018) 242 [arXiv:1907.02341] [InSPIRE].

[105] J. Barrientos et al., Nonminimal couplings, gravitational waves, and torsion in Horndeski's theory, Phys. Rev. D 96 (2017) 084023 [arXiv:1703.09686] [InSPIRE].

[106] J. Barrientos et al., Luminal propagation of gravitational waves in scalar-tensor theories: the case for torsion, Phys. Rev. D 100 (2019) 124039 [arXiv: 1910.00148] [INSPIRE].

[107] A. Golovnev, On the recently proposed mimetic dark matter, Phys. Lett. B 728 (2014) 39 [arXiv: 1310.2790] [INSPIRE]. 
[108] P. Salgado, R.J. Szabo and O. Valdivia, Topological gravity and transgression holography, Phys. Rev. D 89 (2014) 084077 [arXiv:1401.3653] [INSPIRE].

[109] R.J. Szabo and O. Valdivia, Covariant Quiver Gauge Theories, JHEP 06 (2014) 144 [arXiv: 1404.4319] [INSPIRE].

[110] O. Fierro, F. Izaurieta, P. Salgado and O. Valdivia, Minimal AdS-Lorentz supergravity in three-dimensions, Phys. Lett. B $\mathbf{7 8 8}$ (2019) 198 [arXiv:1401.3697] [INSPIRE].

[111] J. Diaz et al., A generalized action for $(2+1)$-dimensional Chern-Simons gravity, J. Phys. A 45 (2012) 255207 [arXiv:1311.2215] [INSPIRE].

[112] A. Cid, F. Izaurieta, G. Leon, P. Medina and D. Narbona, Non-minimally coupled scalar field cosmology with torsion, JCAP 04 (2018) 041 [arXiv: 1704. 04563] [INSPIRE].

[113] J. Barrientos et al., Luminal propagation of gravitational waves in scalar-tensor theories: the case for torsion, Phys. Rev. D 100 (2019) 124039 [arXiv: 1910.00148] [INSPIRE].

[114] A. Toloza and J. Zanelli, Cosmology with scalar-Euler form coupling, Class. Quant. Grav. 30 (2013) 135003 [arXiv: 1301.0821] [INSPIRE].

[115] P. Pani and V. Cardoso, Are black holes in alternative theories serious astrophysical candidates? The case for Einstein-Dilaton-Gauss-Bonnet black holes, Phys. Rev. D 79 (2009) 084031 [arXiv:0902.1569] [INSPIRE].

[116] F.W. Hehl, Four lectures on Poincaré gauge field theory, in Cosmology and gravitation: spin, torsion, rotation, and supergravity, P.G. Bergmann and V. De Sabbata, eds., Plenum Press, New York U.S.A. (1980).

[117] M. Blagojevic, Three lectures on Poincaré gauge theory, SFIN A 1 (2003) 147 [gr-qc/0302040] [INSPIRE].

[118] M. Blagojevic and F.W. Hehl, Gauge theories of gravitation, arXiv:1210.3775 [INSPIRE].

[119] Y.N. Obukhov, Poincaré gauge gravity: an overview, Int. J. Geom. Meth. Mod. Phys. 15 (2018) 1840005 [arXiv: 1805.07385] [inSPIRE].

[120] Y.N. Obukhov and F.W. Hehl, General relativity as a special case of Poincaré gauge gravity, Phys. Rev. D 102 (2020) 044058 [arXiv:2007.00043] [INSPIRE].

[121] T.W.B. Kibble, Lorentz invariance and the gravitational field, J. Math. Phys. 2 (1961) 212 [INSPIRE].

[122] D.W. Sciama, The Physical structure of general relativity, Rev. Mod. Phys. 36 (1964) 463 [Erratum ibid. 36 (1964) 1103] [INSPIRE].

[123] F.W. Hehl and B.K. Datta, Nonlinear spinor equation and asymmetric connection in general relativity, J. Math. Phys. 12 (1971) 1334 [INSPIRE].

[124] F.W. Hehl, P. Von Der Heyde, G.D. Kerlick and J.M. Nester, General relativity with spin and torsion: foundations and prospects, Rev. Mod. Phys. 48 (1976) 393 [INSPIRE].

[125] I.L. Shapiro, Physical aspects of the space-time torsion, Phys. Rept. 357 (2002) 113 [hep-th/0103093] [INSPIRE].

[126] R.T. Hammond, Torsion gravity, Rept. Prog. Phys. 65 (2002) 599 [inSPIRE].

[127] N.J. Poplawski, Classical physics: spacetime and fields, arXiv:0911.0334 [INSPIRE].

[128] N.J. Poplawski, Nonsingular Dirac particles in spacetime with torsion, Phys. Lett. B 690 (2010) 73 [Erratum ibid. 727 (2013) 575] [arXiv:0910.1181] [InSPIRE]. 
[129] W. Kopczyński, A non-singular universe with torsion, Phys. Lett. A 39 (1972) 219 [INSPIRE].

[130] W. Kopczyński, An anisotropic universe with torsion, Phys. Lett. A 43 (1973) 63.

[131] A. Trautman, Spin and torsion may avert gravitational singularities, Nature Phys. Sci. 242 (1973) 7 .

[132] F.W. Hehl, G.D. Kerlick and P. Von Der Heyde, General relativity with spin and torsion and its deviations from Einstein's theory, Phys. Rev. D 10 (1974) 1066 [InSPIRE].

[133] V. De Sabbata and C. Sivaram, Torsion and the cosmological constant problem, Astrophys. Space Sci. 165 (1990) 51.

[134] M. Gasperini, Spin dominated inflation in the Einstein-Cartan theory, Phys. Rev. Lett. 56 (1986) 2873 [INSPIRE].

[135] N. Popławski, The simplest origin of the big bounce and inflation, Int. J. Mod. Phys. D 27 (2018) 1847020 [arXiv:1801.08076] [inSPIRE].

[136] N. Popławski, Universe in a black hole in Einstein-Cartan gravity, Astrophys. J. 832 (2016) 96 [arXiv: 1410.3881] [INSPIRE].

[137] S. Sur, A. Dutta and H.R. Chothe, Mimetic-metric-torsion with induced axial mode and Phantom barrier crossing, arXiv:2007.04906 [INSPIRE].

[138] L. Landau and E. Lifschits, The classical theory of fields, Pergamon Press, Oxford U.K. (1975).

[139] R.J. Bueno Rogerio, J.M. Hoff da Silva, S.H. Pereira and R. da Rocha, A framework to a mass dimension one fermionic $\sigma$-model, EPL 113 (2016) 60001 [arXiv:1603.09183] [INSPIRE].

[140] S.H. Pereira and A. Pinho S. S., ELKO applications in cosmology, Int. J. Mod. Phys. D 23 (2014) 1444008 [INSPIRE].

[141] C.G. Boehmer and J. Burnett, Dark spinors with torsion in cosmology, Phys. Rev. D 78 (2008) 104001 [arXiv:0809.0469] [INSPIRE].

[142] D. Ahluwalia, Mass dimension one fermions, Cambridge University Press, Cambridge U.K. (2019) [arXiv: 2007.15098] [INSPIRE].

[143] D.V. Ahluwalia, Evading Weinberg's no-go theorem to construct mass dimension one fermions: constructing darkness, EPL 118 (2017) 60001 [arXiv: 1605. 04224] [INSPIRE].

[144] D.V. Ahluwalia, The theory of local mass dimension one fermions of spin one half, Adv. Appl. Clifford Algebras 27 (2017) 2247 [arXiv:1601.03188] [INSPIRE].

[145] D.V. Ahluwalia and A.C. Nayak, Elko and mass dimension one field of spin one half: causality and Fermi statistics, Int. J. Mod. Phys. D 23 (2015) 1430026 [arXiv:1502.01940] [INSPIRE]. 